\title{
A note on the $k$-tuple total domination number of a graph
}

\author{
Adel P. Kazemi \\ Department of Mathematics, University of Mohaghegh Ardabili, P. O. Box 5619911367, Ardabil, Iran \\ E-mail: a.p.kazemi@uma.ac.ir, adelpkazemi@yahoo.com
}

\begin{abstract}
For every positive integer $k$ and every graph $G=(V, E)$ with minimum degree at least $k$, a vertex set $S$ is a $k$-tuple total dominating set (resp. $k$-tuple dominating set) of $G$, if for every vertex $v \in V,\left|N_{G}(v) \cap S\right| \geq k$, (resp. $\left|N_{G}[v] \cap S\right| \geq k$ ). The $k$-tuple total domination number $\gamma_{\times k, t}(G)$ (resp. $k$-tuple domination number $\gamma_{\times k}(G)$ ) is the minimum cardinality of a $k$-tuple total dominating set (resp. $k$-tuple dominating set) of $G$.

In this paper, we first prove that if $m$ is a positive integer, then for which graphs $G, \gamma_{\times k, t}(G)=$ $m$ or $\gamma_{\times k}(G)=m$, and give a necessary and sufficient condition for $\gamma_{\times k, t}(G)=\gamma_{\times(k+1)}(G)$. Then we show that if $G$ is a graph of order $n$ with $\delta(G) \geq k+1 \geq 2$, then $\gamma_{\times k, t}(G)$ has the lower bound $2 \gamma_{\times(k+1)}(G)-n$ and characterize graphs that equality holds for them. Finally we present two upper bounds for the $k$-tuple total domination number of a graph in terms of its order, minimum degree and $k$.
\end{abstract}

2010 Mathematics Subject Classification. 05C69.

Keywords. $k$-tuple domination number, $k$-tuple total domination number, $k$-transversal, open neighborhood hypergraph, expectation.

\section{Introduction}

\subsection{Definitions and notations}

All graphs considered here are finite, undirected, and simple. For standard graph theory terminology not given here we refer to [3]. Let $G=(V, E)$ be a graph with vertex set $V$ of order $n(G)=n$ and edge set $E$ of size $m(G)=m$. The minimum and maximum degree of $G$ are respectively denoted by $\delta(G)$ and $\Delta(G)$. The open neighborhood of a vertex $v \in V$ is $N_{G}(v)=\{u \in V \mid u v \in E\}$ and its closed neighborhood is $N_{G}[v]=N_{G}(v) \cup\{v\}$. The degree of a vertex $v$ in $G$ is $\operatorname{deg}(v)=\operatorname{deg}_{G}(v)=1$ $N_{G}(v) \mid$. We write $K_{n}$ and $G[S]$ respectively for the complete graph of order $n$ and the induced subgraph of $G$ by a vertex set $S$.

Let $S \subseteq V$ be a set with cardinality at least $k$. For each $k$-element subset $S^{\prime} \subseteq S$, we define the $(S, k)$-private neighborhood $p n_{k}\left(S^{\prime}, S\right)$ of $S^{\prime}$ to be the set of all vertices $v$ in $V$ such that $N(v) \cap S=S^{\prime}$ (possibly, $v \in S$ ). More exactly, the external $(S, k)$-private neighborhood epn ${ }_{k}\left(S^{\prime}, S\right)$ of $S^{\prime}$ is the set of all vertices $v \in V-S$ such that $N(v) \cap S=S^{\prime}$, and also the inner $(S, k)$-private neighborhood $\operatorname{ipn}_{k}\left(S^{\prime}, S\right)$ of $S^{\prime}$ is the set of all vertices $v \in S$ such that $N(v) \cap S=S^{\prime}$. Further, we define the open $k$-boundary $O B_{k}(S)$ of $S$ to be the set of all vertices $v$ in $G$ such that $v \in p n_{k}\left(S^{\prime}, S\right)$ for some $k$-element subset $S^{\prime} \subseteq S$. Obviously, $O B_{k}(S)=\cup_{S^{\prime}} p n_{k}\left(S^{\prime}, S\right)$, where $S^{\prime}$ is a $k$-element subset of $S$.

Let $k \geq 1$ be an integer. The $k$-join $G \circ_{k} H$ of a graph $G$ to a graph $H$ of order at least $k$ is the graph obtained from the disjoint union of $G$ and $H$ by joining each vertex of $G$ to at least $k$ vertices of $H$. Also for any two graphs $G$ and $H$ the corona of $H$ and $G$ is the graph $H \circ G$ formed from one copy of $H$ and $|V(H)|$ copies of $G$ where the $i$-th vertex of $H$ is adjacent to every vertex in the $i$-th copy of $G$. 


\subsection{Domination}

Domination in graphs is now well studied in graph theory and the literature on this subject has been surveyed and detailed in the two books by Haynes, Hedetniemi, and Slater [3, 4]. A set $S \subseteq V$ is a dominating set if each vertex in $V \backslash S$ is adjacent to at least one vertex of $S$. The domination number $\gamma(G)$ is the minimum cardinality of a dominating set. A set $S \subseteq V$ is a total dominating set if each vertex in $V$ is adjacent to at least one vertex of $S$, while the minimum cardinality of a total dominating set is the total domination number $\gamma_{t}(G)$ of $G$. The reader can see [5] for more details on total domination number.

In [2] Harary and Haynes defined a generalization of domination as follow. Let $k \geq 1$ be an integer. A subset $S$ of $V$ is a $k$-tuple dominating set, abbreviated $\mathrm{kDS}$, of $G$ if for every vertex $v \in V,\left|N_{G}[v] \cap S\right| \geq k$, that is, $v$ is in $S$ and has at least $k-1$ neighbors in $S$ or $v$ is in $V-S$ and has at least $k$ neighbors in $S$. The $k$-tuple domination number $\gamma_{\times k}(G)$ of $G$ is the minimum cardinality of a kDS of $G$. Clearly, $\gamma(G)=\gamma_{\times 1}(G) \leq \gamma_{\times k}(G) \leq \gamma_{\times(k+1)}(G)$, while $\gamma_{t}(G) \leq \gamma_{\times 2}(G)$. For a graph to have a $\mathrm{kDS}$, its minimum degree is at least $k-1$. Hence for trees, $k \leq 2$. A $k$-tuple dominating set where $k=2$ is called a double dominating set (abbreviated DDS). The concept of $k$-tuple domination has been studied by several authors (see, for example, [11, 12], and elsewhere).

In [6], Henning and Kazemi started the studying of $k$-tuple total domination number in graphs, which is an extention of total domination number. For an integer $k \geq 1$, a subset $S$ of $V$ is a $k$-tuple total dominating set of $G$, abbreviated kTDS, if for every vertex $v \in V,|N(v) \cap S| \geq k$; that is, $S$ is a kTDS if every vertex has at least $k$ neighbors in $S$. The $k$-tuple total domination number $\gamma_{\times k, t}(G)$ is the minimum cardinality of a kTDS of $G$. A kTDS $S$ in a graph $G$ is minimal if no proper subset of $S$ is a kTDS in $G$. We remark that $\gamma_{t}(G)=\gamma_{\times 1, t}(G)$. For a graph to have a kTDS, its minimum degree is at least $k$. Since every $(\mathrm{k}+1)$ TDS is also a kTDS, we note that $\gamma_{\times k, t}(G) \leq \gamma_{\times(k+1), t}(G)$ for all graphs with minimum degree at least $k+1$. A kTDS of cardinality $\gamma_{\times k, t}(G)$ is called a $\gamma_{\times k, t}(G)$-set. When $k=2, k$-tuple total dominating set and $k$-tuple total domination number are known respectively as double total dominating set, abbreviated DTDS, and double total domination number. For more information about the concept of $k$-tuple domination see $[6,7,8]$.

\subsection{Our goal}

In this paper, in Section 2, we will prove that if $m$ is a positive integer, then for which graphs $G$, $\gamma_{F}(G)=m$ where $\gamma_{F}(G)$ is $\gamma_{\times k, t}(G)$ or $\gamma_{\times k}(G)$. Then we will present a necessary and sufficient condition for $\gamma_{\times k, t}(G)=\gamma_{\times(k+1)}(G)$. In Section 3, we will show that if $G$ is a graph of order $n$ with $\delta(G) \geq k+1 \geq 2$, then $\gamma_{\times k, t}(G)$ has the lower bound $2 \gamma_{\times(k+1)}(G)-n$ and characterize graphs that equality holds for them. Finally, in Section 4, two upper bounds for the $k$-tuple total domination number of a graph is given in terms of its order and minimum degree.

The following propositions are useful in the context.

Proposition 1.1. (Henning, Kazemi [6] 2010) Let $G$ be a graph with $\delta(G) \geq k$, and let $S$ be a kTDS of $G$. Then

a. $\max \left\{\gamma_{\times k}(G), k+1\right\} \leq \gamma_{\times k, t}(G) \leq \gamma_{\times(k+1)}(G)$,

b. for each spanning subgraph $G$ of a graph $H, \gamma_{\times k, t}(H) \leq \gamma_{\times k, t}(G)$,

c. the open neighorhood of each vertex of degree $k$ is a subset of $S$.

Proposition 1.2. (Henning, Kazemi [6] 2010) Let $G$ be a graph of order $n$ with $\delta(G) \geq k$, and let $S$ be a kTDS of $G$. Then $S$ is minimal if and only if 
a. $S \backslash\{x\}$ is not a kTDS for every $x \in S$, or

b. for each vertex $x \in S$, there exists a $k$-element subset $S_{x} \subseteq S$ such that $x \in S_{x}$ and $\left|p n_{k}\left(S_{x}, S\right)\right|=$ $\left|\operatorname{epn}_{k}\left(S_{x}, S\right)\right|+\left|i p n_{k}\left(S_{x}, S\right)\right| \geq 1$, or

c. $O B_{k}(S)$ dominates $S$.

Proposition 1.3. (Henning, Kazemi [6] 2010) Let $G$ be a graph with $\delta(G) \geq k$. Then, $\gamma_{\times k, t}(G)=k+1$ if and only if $G=K_{k+1}$ or $G=F \circ_{k} K_{k+1}$ for some graph $F$.

Proposition 1.4. (Haynes, Hedetniemi, Slater [3] 1998) For a graph $G$ with even order $n$ and no isolated vertices, $\gamma(G)=\frac{n}{2}$ if and only if the components of $G$ are the cycle $C_{4}$ or the corona $H \circ K_{1}$ for any connected graph $H$.

Proposition 1.5. (Henning, Yeo, [9] 2007) If $G$ is a graph of order $n$ and minimum degree $\delta \geq 1$, then

$$
\gamma_{t}(G) \leq\left(\frac{1+\ln \delta}{\delta}\right) n .
$$

\section{$2 \quad$ When $\gamma_{\times k, t}(G)=\gamma_{\times(k+1)}(G)$ ?}

Before we characterize the graphs $G$ that satisfy $\gamma_{\times k, t}(G)=\gamma_{\times(k+1)}(G)$, we need to recognize $\gamma_{\times k, t}(G)$ and $\gamma_{\times(k+1)}(G)$, for any graph $G$. For finding $\gamma_{\times k, t}(G)=m$, we consider $m \geq k+2$, by Proposition 1.3.

Theorem 2.1. Let $G$ be a graph with $\delta(G) \geq k \geq 1$. Then for any integer $m \geq k+2, \gamma_{\times k, t}(G)=m$ if and only if $G=K_{m}^{\prime}$ or $G=F \circ_{k} K_{m}^{\prime}$, for some graph $F$ and some spanning subgraph $K_{m}^{\prime}$ of $K_{m}$ with $\delta\left(K_{m}^{\prime}\right) \geq k$ such that $m$ is minimum in the set

$$
\left\{t \mid G=F \circ_{k} K_{t}^{\prime} \text {, for some } F \text { and some spanning subgraph } K_{t}^{\prime} \text { of } K_{t}, \delta\left(K_{t}^{\prime}\right) \geq k\right\} \text {. }
$$

Proof. Let $S$ be a $\gamma_{\times k, t}(G)$-set and let $\gamma_{\times k, t}(G)=m$, for some $m \geq k+2$. Then $|S|=m$, and every vertex has at least $k$ neighbors in $S$. Hence $G[S]=K_{m}^{\prime}$, for some spanning subgraph $K_{m}^{\prime}$ of $K_{m}$ and $\delta\left(K_{m}^{\prime}\right) \geq k$. If $|V|=m$, then $G=K_{m}^{\prime}$. If $|V|>m$, let $F=G[V-S]$. Since every vertex in $V-S$ has at least $k$ neighbors in $S$, we obtain $G=F \circ_{k} K_{m}^{\prime}$. Also by the definition of $k$-tuple total domination number, clearly $m$ is the minimum of the set given in (2.1).

Conversely, let $G=K_{m}^{\prime}$ or $G=F \circ_{k} K_{m}^{\prime}$, fo some graph $F$ and some spanning subgraph $K_{m}^{\prime}$ of $K_{m}$ with $\delta\left(K_{m}^{\prime}\right) \geq k$ such that $m$ is the minimum of the set given in (2.1). Then, since $V\left(K_{m}^{\prime}\right)$ is a kTDS of $G$ with cardinality $m, \gamma_{\times k, t}(G) \leq m$. Now we prove $\gamma_{\times k, t}(G)=m$. For $m=k+1$, it holds, by Proposition 1.3. Now let $m \geq k+2$. If $\gamma_{\times k, t}(G)=m^{\prime}$, for some $m^{\prime}<m$, then the previous paragraph shows that for some graph $F^{\prime}$ and some spanning subgraph $K_{m^{\prime}}^{\prime}$ of $K_{m^{\prime}}$ with $\delta\left(K_{m^{\prime}}^{\prime}\right) \geq k$, $G=F^{\prime} \circ_{k} K_{m^{\prime}}^{\prime}$ contradicting with the minimality of $m$. Therefore $\gamma_{\times k, t}(G)=m$.

Q.E.D.

Similar to the proof of Theorem 2.1, we can prove next theorem.

Theorem 2.2. Let $G$ be a graph with $\delta(G) \geq k$. Then for any integer $m \geq k+1, \gamma_{\times(k+1)}(G)=m$ if and only if $G=K_{m}^{\prime}$ or $G=F \circ_{k+1} K_{m}^{\prime}$, for some graph $F$ and some spanning subgraph $K_{m}^{\prime}$ of $K_{m}$ with $\delta\left(K_{m}^{\prime}\right) \geq k$ such that $m$ is minimum in the set

$$
\left\{t \mid G=F \circ_{k+1} K_{t}^{\prime} \text {, for some } F \text { and some spanning subgraph } K_{t}^{\prime} \text { of } K_{t}, \delta\left(K_{t}^{\prime}\right) \geq k\right\} .
$$


The next theorem can be obtained by Propositions 1.2(b), 1.3, and Theorems 2.1, 2.2.

Theorem 2.3. For every graph $G$ with $\delta(G) \geq k, \gamma_{\times k, t}(G)=\gamma_{\times(k+1)}(G)$ if and only if $G$ contains a $\gamma_{\times k, t}(G)$-set $S$ such that $O B_{k}(S) \subseteq S$.

\section{A lower bound}

Here we give a lower bound on the $k$-tuple total domination number of a graph in terms of its order and $(k+1)$-tuple domination number.

Theorem 3.1. Let $G$ be a graph of order $n$ with $\delta(G) \geq k+1 \geq 2$. Then

$$
\gamma_{\times k, t}(G) \geq 2 \gamma_{\times(k+1)}(G)-n
$$

Proof. Let $S$ be a $\gamma_{\times k, t}(G)$-set and let $A$ be the set of all isolated vertices in $G[V(G)-S]$. Then $G[V(G)-(S \cup A)]$ contains no isolated vertices. Let $D$ be a minimum dominating set of $G[V(G)-$ $(S \cup A)]$. Then the well-known inequality of Ore [10] implies

$$
\begin{aligned}
|D| & \leq \frac{|V(G)-(S \cup A)|}{2} \\
& \leq \frac{|V(G)-S|}{2} \\
& =\frac{n-\gamma_{\times k, t}(G)}{2}
\end{aligned}
$$

Since $\delta(G) \geq k+1$, and $S$ is a $\operatorname{kTDS}$ of $G$, we conclude that $S \cup D$ is a $(k+1)$-tuple dominating set of $G$, and so

$$
\begin{aligned}
\gamma_{\times(k+1)}(G) & \leq|S \cup D| \\
& =\gamma_{\times k, t}(G)+|D| \\
& \leq \frac{n+\gamma_{\times k, t}(G)}{2} .
\end{aligned}
$$

Q.E.D.

We notice that $\gamma_{\times k, t}(G)=2 \gamma_{\times(k+1)}(G)-n$ if and only if every inequality in the proof of Theorem 3.1 is an equality. But this holds if and only if $A=\varnothing,|S \cup D|=\gamma_{\times(k+1)}(G)$ and $|D|=\frac{n-\gamma_{\times k, t}(G)}{2}$. Thus $\gamma_{\times k, t}(G)=2 \gamma_{\times(k+1)}(G)-n$ if and only if $\delta(G-S) \geq 1, O B_{k}(S) \nsubseteq S$ and (by Proposition 1.4) the components of $G-S$ are the cycle $C_{4}$ or the corona $H \circ K_{1}$ for any connected graph $H$. Therefore we have proved next theorem.

Theorem 3.2. Let $G$ be a graph of order $n$ with $\delta(G) \geq k+1 \geq 2$. Then

$$
\gamma_{\times k, t}(G)=2 \gamma_{\times(k+1)}(G)-n
$$

if and only if $\gamma_{\times k, t}(G)=n$ or there exists a $\gamma_{\times k, t}(G)$-set $S$ such that $\delta(G-S) \geq 1, O B_{k}(S) \nsubseteq S$ and the components of $G-S$ are the cycle $C_{4}$ or the corona $H \circ K_{1}$ for any connected graph $H$.

For example, if $G$ is a $k$-regular graph of order $n$, then

$$
2 \gamma_{\times(k+1)}(G)-n=\gamma_{\times k, t}(G)=\gamma_{\times(k+1)}(G)=n,
$$

and if $G$ is the complete graph $K_{n}$ of order at least $k+2$, then

$$
2 \gamma_{\times(k+1)}(G)-n<\gamma_{\times k, t}(G)=\gamma_{\times(k+1)}(G)=k+1 .
$$




\section{Two upper bounds}

Hypergraphs are systems of sets which are conceived as natural extensions of graphs. A hypergraph $H=(V, C)$ is a finite set $V$ of elements, called vertices, together with a finite multiset $C$ of arbitrary subsets of $V$, called edges. The degree of a vertex $v$ in $H$, denoted by $\operatorname{deg}(v)$ or $\operatorname{deg}_{H}(v)$, is the number of edges that contain $v$. A $k$-uniform hypergraph is a hypergraph in which every edge has size $k$. Every simple graph is a 2-uniform hypergraph. Thus graphs are special hypergraphs.

For each positive integer $k$, Henning and Kazemi [6] defined the concept of $k$-transversal numer in hypergraphs as follows: the $k$-transversal number $\tau_{k}(H)$ of a hypergraph $H=(V, E)$ is the minimum size of a $k$-transversal in $H$, that is, a subset $S \subseteq V$ such that contains at least $k$ vertices of every edge in $H$. Recall that $\tau_{1}(H)$ is known as transversal number $\tau(H)$ of $H$.

For a graph $G=(V, E)$, we denote by $H_{G}$ the open neighborhood hypergraph of $G$; that is, $H_{G}=(V, C)$ is the hypergraph with vertex set $V$ and with edge set $C$ consisting of the open neighborhoods of vertices of $V$ in $G$.

We recall the next proposition from [6].

Proposition 4.1. (Henning, Kazemi [6] 2010) If $G$ is a graph with minimum degree at least $k$ and $H_{G}$ is the open neighborhood hypergraph of $G$, then $\gamma_{\times k, t}(G)=\tau_{k}\left(H_{G}\right)$.

Perhaps much of the recent interest in total domination in graphs arises from the fact that total domination in graphs can be translated to the problem of finding transversals in hypergraphs. Proposition 4.1 shows that the problem of finding $k$-tuple total dominating sets in graphs can be translated to the problem of finding $k$-transversals in hypergraphs. The main advantage of considering hypergraphs rather than graphs is that the structure is easier to handle-for example, we can often restrict our attention to uniform hypergraphs where every edge has the same size.

Let $H=(V, C)$ be a hypergraph and let $V_{k} \subseteq V$ be a subset of vertices with cardinality $k$. We define $d_{k}\left(V_{k}\right)$ as the number of edges $e$ of $H$ such that $V_{k} \subseteq e$, and also

$$
\Delta_{k}(H)=\max \left\{d_{k}\left(V_{k}\right) \mid V_{k} \subseteq V\right\} .
$$

Notice that $d_{1}\left(V_{1}\right)=\operatorname{deg}_{H}(v)$, where $V_{1}=\{v\}$, and $\Delta_{1}(H)$ is the same maximum degree $\Delta(H)$ among the vertices of $H$. The components of a hypergraph $H$ are the minimal sets of vertices in $H$ such that no edge in $H$ contains vertices from different components. A hypergraph is called connected if it contains one component. For disjoint subsets $X$ and $Y$ of vertices in a hypergraph $H$, we define $H(X, Y)$ to be the hypergraph obtained from $H$ by deleting the vertices in $X$ and all edges incident with $X$ and by deleting the vertices in $Y$ and shrinking all edges incident with $Y$. Furthermore, if $Y=\varnothing$, we denote $H(X, Y)$ simply by $H-X$ or by $H-v$ if $X=\{v\}$.

In the next two theorems we will present two upper bounds on the $k$-tuple total domination number of a graph $G$ in terms of its order, minimum degree and $k$. The proof of the following is along similar lines to the proof presented in Proposition 1.5 by Henning and Yeo for the total domination number, and thus we omit it.

Theorem 4.2. Let $G$ be a graph of order $n$ with $\delta(G) \geq k \geq 1$. If for every two vertices $\mathrm{x}$ and $\mathrm{y}$ of $\mathrm{G}, N_{G}(x) \cap N_{G}(y)=\varnothing$ or $\left|N_{G}(x) \cap N_{G}(y)\right| \geq k$, then

$$
\gamma_{\times k, t}(G) \leq\left(\frac{1+\ln \delta}{\delta}\right)\left(\begin{array}{l}
n \\
k
\end{array}\right) .
$$


Before we present the second upper bound, we define some needed notations. Suppose that $G$ is a graph with the vertex set $V(G)=\left\{v_{1}, v_{2}, \ldots, v_{n}\right\}$ and every vertex $v_{i}$ has degree $d_{i}$. For any integer $m$, we define

$$
\widehat{d_{m}}=\frac{1}{n} \sum_{i=1}^{n}\left(\begin{array}{l}
d_{i} \\
m
\end{array}\right) .
$$

By these notations, we present next theorem, which its proof is along similar lines to the presented by Chang [1] for the $k$-tuple domination number, and thus we omit the proof.

Theorem 4.3. If $k$ is a positive integer and $G$ is a graph of order $n$ with $\delta>k \geq 1$, then

$$
\gamma_{\times k, t}(G) \leq\left(\frac{\ln (\delta-k)+\ln \hat{d_{k}}+1}{\delta-k}\right) n .
$$

\section{References}

[1] G. Chang, The upper bound on k-tuple domination numbers of graphs, European Journal of Combinatorics 29 (2008) 1333-1336.

[2] F. Harary, T. W. Haynes, Double domination in graphs, Ars Combin. 55 (2000) 201-213.

[3] T. W. Haynes, S. T. Hedetniemi, P. J. Slater (eds). Fundamentals Domination in Graphs, Marcel Dekker, Inc. New York, 1998.

[4] T. W. Haynes, S. T. Hedetniemi, P. J. Slater (eds), Domination in Graphs: Advanced Topics, Marcel Dekker, Inc. New York, 1998.

[5] M. A. Henning, A survey of selected recent results on total domination in graphs, Discrete Math., 309 (2009) 32-63.

[6] M. A. Henning, A. P. Kazemi, k-tuple total domination in graphs, Discrete Applied Math. 158 (2010) 1006-1011.

[7] M. A. Henning, A. P. Kazemi, $k$-tuple total domination in cross product of graphs, J Comb. Optim. 24 (2012) 339-346.

[8] M. A. Henning, A. P. Kazemi, Total domination in inflated graphs, Discrete Applied Mathematics 160 (2012) 164-169.

[9] M. A. Henning, A. Yeo, A transition from total domination in graphs to transversals in hypergraphs, Quaestiones Mathematicae 30 (2007) 417-436.

[10] O. Ore, Theory of Graphs, vol. 38, Amer. Math. Soc. Colloq. Publ., 1962.

[11] C. S. Liao, G. J. Chang, Algorithmic aspects of k-tuple domination in graphs, Taiwanese J. Math. 6 (2002) 415-420.

[12] C. S. Liao, G. J. Chang, k-tuple domination in graphs, Information Processing Letters 87 (2003) 45-50. 